\title{
AN ANNOTATION TOOL FOR BENCHMARKING METHODS FOR AUTOMATED CONSTRUCTION WORKER POSE ESTIMATION AND ACTIVITY ANALYSIS
}

\author{
D. Roberts ${ }^{* 1}$, M. Wang ${ }^{2}$, W. Torres Calderon ${ }^{1}$ and M. Golparvar-Fard ${ }^{1}$ \\ ${ }^{1}$ University of Illinois at Urbana-Champaign, Champaign, USA \\ ${ }^{2}$ Hong Kong University of Science and Technology, Hong Kong, China \\ * Corresponding author
}

\begin{abstract}
The increase in affordability and quality of jobsite cameras provides opportunities to facilitate monitoring of construction worker activities. Research has highlighted the potential of computer vision algorithms to be used for automated activity analysis. However, applying and adapting such techniques to the construction setting and deploying them for use on job sites entails extensive validation against a construction-specific benchmark dataset. Most state-of-the-art computer vision algorithms are strongly supervised and thus such benchmarks require large amounts of per-image ground truth annotations, both to enable the algorithm to learn how to perform its task during training time and for evaluation on a separate portion of this dataset at test-time. We address this need by introducing an annotation tool that enables users to label visual footage of construction workers by positioning both worker instances and individual key points corresponding to the body parts of each worker (e.g., left wrist, right elbow, nose) in the scene. This annotation tool is the first such publicly available tool capable of producing ground truth for pose estimation, tracking and activity analysis methods to the best of our knowledge. We demonstrate the capabilities of our tool by exhaustively annotating key points, identities and activity labels in a dataset of 393 image sequences that depict workers performing various construction-related activities. This preliminary set of annotation tasks demonstrates the ease of use and flexibility of our annotation tool.
\end{abstract}

\section{Introduction}

Ensuring the workface operates at high levels of efficiency while conforming to safety standards is one of the top priorities of project managers on construction sites. Today, measuring and improving productivity of a construction crew is determined via activity analysis (Gouett et al., 2011). This typically involves the elaboration of crew-balance charts from reports provided by a superintendent observing teams of workers or equipment. Likewise, safety monitoring is performed by designated observers that monitor worker ergonomics and check for unsafe proximity to hazardous objects or areas. These two aspects of workface assessment are thus subject to the human error inherent to manual inspection that can arise due to issues such as fatigue. These observations motivate the task of automating these two processes, which has been the focus of considerable research efforts. Various types of sensorial data have been used to this effect, such as GPS, Ultra-Wide Band (Cheng et al., 2011) and in the case of construction equipment, telematics (Monnot and Williams, 2011). The availability of highly affordable and high-quality cameras over the past decade has brought about their increased usage on jobsites and highlighted the potential of decision making based on visual data. Judiciously positioned static cameras can be used for streaming footage of worker operations. Unmanned Aerial Vehicles (UAVs) have also become a popular means of determining construction progress (Ham et al., 2016) and of envisioning construction operations from otherwise inaccessible camera viewpoints. Visual data has thus attracted considerable attention from the construction community as a viable data source from which safety monitoring and activity analysis can be conducted. Furthermore, deep learning-based computer vision algorithms have recently shown great promise for obtaining detailed semantic understanding of visual scenes (Lecun et al., 2015).

Particularly relevant to the construction setting is progress made in the domains of human pose estimation and activity segmentation. The objective of the human pose estimation task is to automatically pinpoint the location of each limb of any human present in either $2 \mathrm{D}$ or 3D. On the other hand, activity segmentation aims to determine per-frame activities of humans in site videos and often performs an intermediary pose estimation step. State-of-the-art techniques have shown to be effective at accurately determining human pose and activities in several established benchmarks such as Microsoft COCO (Lin et al., 2014). This hints at their potential for being used in an off-the-shelf fashion on jobsites to determine construction worker pose that can in turn be used to inform verification of conformity to safety regulations or action segmentation algorithms. Conformity with safety regulations could be accomplished by the initial steps of a) recording construction workers with a multiple-camera setup, b) running a 2D worker pose estimator on the resulting video feeds, c) determining the $3 \mathrm{D}$ pose of construction workers by triangulating (Konstantinou and Brilakis, 2016) their 2D positions across camera feeds. This $3 \mathrm{D}$ representation can determine proximity with potentially hazardous objects or zones. If the working 
environment is a jobsite with a corresponding Building Information Model (BIM), a point cloud representation of the jobsite can be created using Structure From Motion (SfM) on sets of images obtained by mobile cameras such as UAVs and aligned with the BIM (Seo et al.., 2015). Video feeds of workers can be registered to this point cloud, effectively localizing them within BIM (Asadi and Han, 2018) whose zones and elements contain corresponding safety-related information. This can be queried in case of worker proximity with a potentially hazardous zone to provide actionable feedback to construction safety practitioners. In addition, 2D pose is highly descriptive of construction worker activity and can be thus used to inform automated vision-based activity analysis methods. These two use cases of $2 \mathrm{D}$ pose estimation methods motivate the usage of such methods in workflows integrating automated safety monitoring and activity analysis.

Crucial to the development of a framework using 2D pose estimation in this manner is the reliability of $2 \mathrm{D}$ pose estimation methods. Their potency across a wide range of visually distinctive settings such as Microsoft COCO suggest they might perform well in construction-specific scenarios. However, prior to definitive deployment of such algorithms on job sites, it is necessary to validate their performance on a benchmark set of construction-specific visual environments to ascertain their potential for generalizing effectively to novel jobsites. Such a benchmark should test the robustness of algorithms to visual challenges specific to the construction setting, among which occlusion and cluttered background figure prominently. To the best of our knowledge, no such benchmark exists. Motivated by this observation, in this work we introduce both an annotation tool and a set of annotations of visual footage of workers performing various construction activities. We show the broad range of visual environments depicted in these videos justify its usage as a benchmark. Our annotation tool enables annotation of construction worker 2D pose and specification of whether individual keypoints are occluded, by background objects or self-occlusion.

\section{Related work}

\subsection{Visual detection and tracking of construction resources}

Localization and tracking of construction resources from visual data has been primarily accomplished at the level of the bounding box. In the computer vision literature bounding-box level detection is known as object detection. These methods have been applied to various types of construction resources such as earthmoving equipment (Memarzadeh et al., 2013; Rezazadeh Azar and McCabe, 2012) and cranes though construction workers have been a notable research focus. (Park and Brilakis, 2012) performed object detection via Histogramof-Gradient (HOG) features for detecting and tracking construction workers. More recent work has leveraged deeplearning based architectures for worker detection (Son et al., 2019) and for cranes (Roberts and Golparvar-Fard, 2017). Only recently have methods attempted to obtain more nuanced representations of construction resources. (Bao et al., 2016) learnt separate HOG-informed object detectors for excavator buckets, carriages and boom-arm joints. (Soltani et al., 2017) learnt object detectors for synthetically generated images depicting individual components of 3D models projecting into 2D. These methods provide a more complete description of the construction resource but require object detectors to be trained for each individual component which is not scalable. The newfound potency of deep learning-based pose estimation methods has found use cases in worker ergonomic assessment (Liu et $a l ., 2017)$. In this work, however, determining the potency of the pose estimation step was not the primary focus of this work. Pose estimation has also been used to determine 2D excavator pose (Xu and Yoon, 2019) from images of excavators.

\subsection{Vision-based activity analysis of construction resources}

Some work on recognizing construction resource activities has been achieved without the help of intermediary representations of the resources in question. Examples of this are (Yang et al., 2016) in which a method based on dense trajectories (Wang et al., 2011) was introduced for classifying videos of construction workers performing actions. More recent works have focused on activity recognition methods relying on resource information. (Khosrowpour et al., 2014) also classified individual videos of construction workers performing drywallrelated operations using RGB and depth data, the latter of which was acquired from a Microsoft Kinect device. (Roberts et al. 2018) and (Bao et al., 2016) introduced methods for determining per-frame activities of construction equipment from long-form videos of earthmoving operations, using perequipment and per-equipment part bounding box information respectively. Kim et al. 2018) performed interaction analysis of earthmoving equipment via bounding box information.

\subsection{Safety monitoring of construction resources}

Previous work has highlighted the potential application of techniques mentioned in the construction safety monitoring of this article. (Seo et al., 2015) noted that identification of resource activity recognition could be used to identify postures and acts that do violate safety rules. Much effort has been invested in investigating methods for automating aspects of vision-based safety inspection such as hard-hat detection (Park et al., 2015). Recent work has explored methods of integrating outputs of vision-based detection methods with parsed safety rules to automatically detect for violations of safety standards (Tang and Golparvar-Fard, 2017) such as those defined by Occupational Safety and Health administration) in the USA.

\subsection{Visual data annotation and benchmarking}

In the computer vision community, the increased performance of deep learning-based scene recognition methods (Lecun et $a l ., 2015)$ have spearheaded research efforts into supervised learning algorithms that can automatically detect and characterize objects and their behaviour in more and more detail. Accompanying the success of these methods are the increased size of the datasets and pertaining annotations. The vast size of datasets such as Microsoft COCO and ImageNet (Russakovsky et al., 2015) has incentivized investigations into streamlining the annotation procedure that has led to 
development of partially automated, human-in-the-loop annotation methods (Castrejón et al., 2017). The vast scale of annotated data has also led to work in on diagnosing error in object detection (Hoiem et al., 2012) and pose estimation methods (Ronchi and Perona, 2017) who provide detailed analysis of types and frequency of errors related to the supervised learning task. In the construction setting, efforts have also been made to crowdsource the annotation procedure (Liu and Golparvar-Fard, 2015) by assigning annotation tasks to Amazon Mechanical Turk (AMT) annotators online who were compensated financially. Object detection benchmarks for construction equipment were introduced in (Kim et al., 2016) with detailed bounding box information for road rollers, concrete mixer trucks, loaders, excavators and dump trucks. Such a benchmark for construction workers has yet to be introduced.

\section{Method}

\subsection{Development of the annotation tool}

We expand on the Computer Vision Annotation Tool (CVAT) re-implementation of Video Annotation Tool from Irvine, California (VATIC), introduced in (Vondrick et. al, 2013). This tool is developed using the Django web development framework which interfaces a PostgreSQL database with a Python back-end and a Javascript front-end. At the time of development this tool enabled users to label objects in uploaded visual data with bounding boxes and specify box categories and other attributes. We modify the functionalities of this annotation tool to instead allow labelling of worker limbs via overlaying "skeletons" on the image and clicking and dragging skeleton keypoints (e.g. nose, left hand, left wrist). The first developmental step involved extending the PostgreSQL database schema to include tables containing skeleton and keypoint identity and position. We then proceeded to adapt the front-end to enable creation, placement and deletion of skeletons as well as editing keypoints of newly created skeletons.

\subsection{Usage}

Our annotation tool is hosted on a webserver that can be accessed via the Virtual Private Network (VPN) of the University of Illinois campus. Users can register for the annotation tool and sign into a tool via a login page, though only users with admin access can upload videos and create and assign annotation tasks. As shown in Figure 1, users can create worker skeletons in the video they are annotating by clicking on the button at the bottom-right of the screen and review annotations present in the videos using the right column. Worker skeletons are composed of 13 keypoints: nose, left/right shoulders, elbows, wrists, hips, knees and ankles. They can edit individual keypoint positions by clicking and dragging the pertaining blue circle on their worker's skeleton or translate the entire skeleton by clicking and dragging on the green circle. A skeleton is automatically created with all its keypoints. If some keypoints are occluded, the user can specify this by hovering the mouse cursor over the keypoint and pressing a hotkey. Before placing a skeleton, it is also possible to flip the orientation of the skeleton via a different hotkey.

Figure 1: User interface of our annotation tool

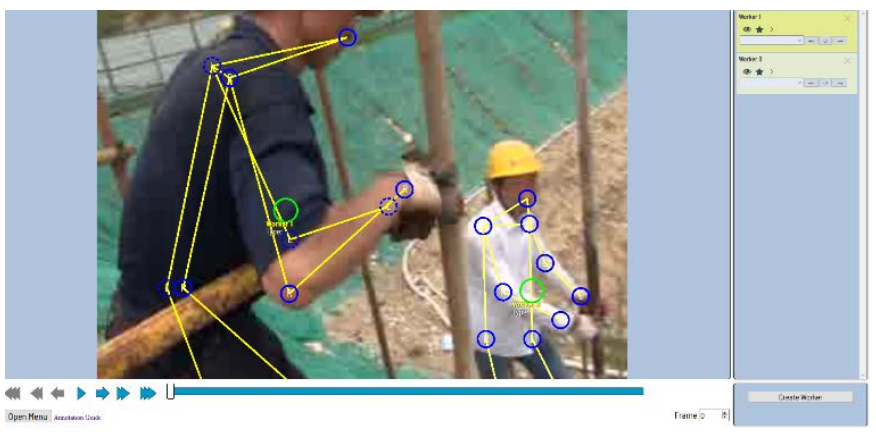

Using the video-style play, forward and backward buttons at the bottom of the screen, users can traverse the worker video and annotate worker position at each video frame. When users move to a previously unannotated video frame, the skeletons on the most recent previously annotated video frame transfer automatically to the new frame. This allows users to exploit the small frame-by-frame skeleton variations to rapidly annotate new frames. In addition, our tool automatically linearly interpolates keypoint positions between keyframes. A keyframe is any frame whose annotations have been manually modified. Users are thus suggested to annotate longer videos in the following manner:

- Choose a frame interval over which the worker keypoints exhibit linear movement (e.g. $\mathrm{n}=15$ frames) and annotate every nth frame in the video starting from frame 0

- Review the annotations and if keypoint positions are inaccurate, repeat the process starting from a frame in between 0 and n (e.g. 11).

- Repeat first two steps as necessary to ensure accurate keypoint localization throughout the video.

When a keypoint is annotated as being occluded, the occluded property automatically carries over to the next keyframe. Only keyframes are saved to the database in order to minimize storage costs, and interpolation is automatically re-applied upon reloading the annotation.

\section{Validation}

\subsection{Dataset}

We annotate 393 videos that were introduced in (Yang et al., 2016). Each video depicts one or two workers performing a construction-related activity, possibly containing more that are watching or performing background activities. The activity types are bricklaying, transporting, cutting plate, drilling, tying rebar, nailing, plastering, shovelling, bolting, welding and sawing. Most workers in the "tying rebar" videos are highly truncated we do not attempt to annotate these videos. Many of the videos in a same activity category are also very visually 
similar as they depict the same construction worker in the same background. Figure 2 shows the rich variety in pose appearance, background, clothing and activity across dataset videos. We note that these videos were captured by jobsite managers with consent of the workers. They are only used for worker training and demonstration of the potential of our method, and not to assess worker performance.

Figure 2. Some example images from the annotated dataset

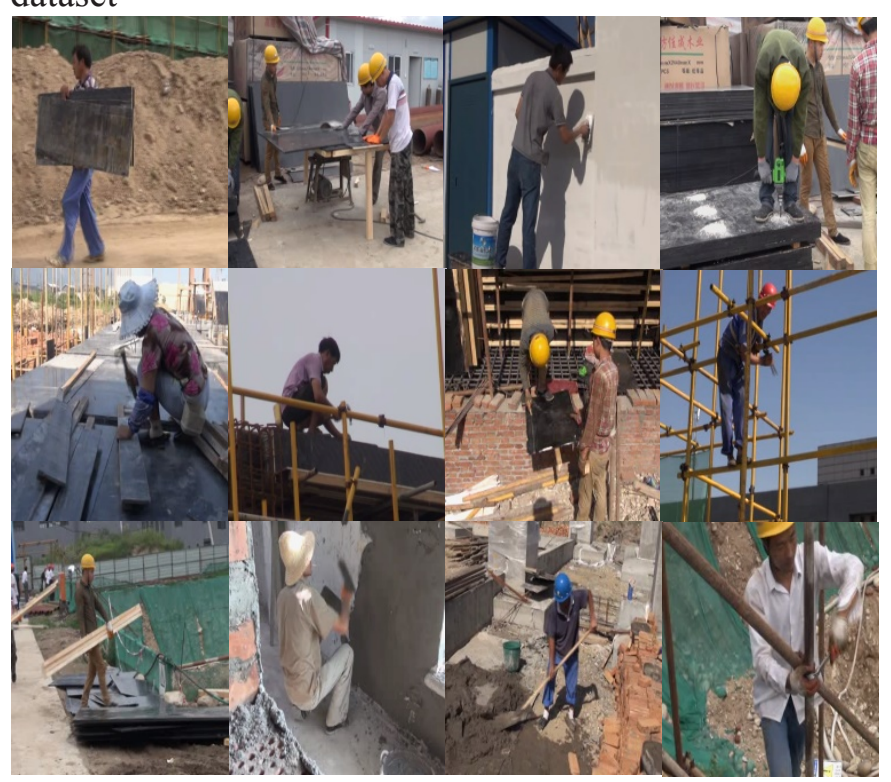

\subsection{Annotation procedure}

We divide the videos among 71 annotators with prior experience in construction management. Each annotator is assigned a roughly equal amount of data as measured by video size in MB (megabytes). This is accomplished by solving the partition problem (Hayes, 2002) using a dynamic programming formulation. Annotators are taught best practices and quality standards via guideline images such as the one in Figure 3 and demo videos and are given intermediary feedback to address concerns with their annotations during the exercise. We impose the convention that the "left" side of a worker's body should correspond to the worker's left side. Annotators are assigned a "Poor", "Quality" or "High Quality" annotation grade based on the localization of the keypoints and plausibility of the specification of the occluded property. An example of a high-quality annotation can be found in Figure 3. In this image, the entire left side of the worker's body except the shoulder is correctly labelled as being occluded either due to the background object (left hip, knee and ankle) or due to self-occlusion (left wrist and elbow). Precisely locating occluded keypoints on a frame-by-frame basis is an ill-defined task. However, their approximate location can generally be inferred by their location in neighbouring frames.
Figure 3. A high-quality annotation

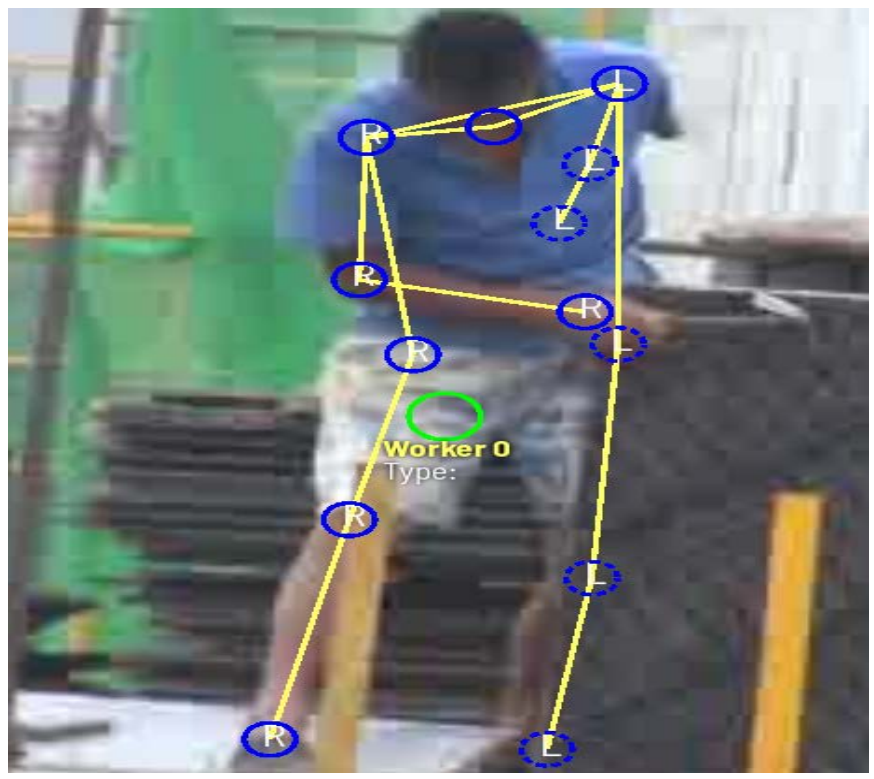

\section{Results}

\subsection{Quality of annotations}

"Poor" annotations were mainly due to inaccurate localization of keypoints stemming from insufficient iterations of the annotation method outlined in 3.2 and non-specification of the occluded property in the scenario of self-occlusion. An additional issue was annotators forgetting to follow the convention concerning the left-right worker orientation. "Quality" annotation keypoints were generally well-localized but suffered from some localization or occlusion-related issues. From a practical point of view, the authors considered the "High Quality" annotations to be usable as is for pose estimation validation purposes and the "Quality" annotations as requiring further work to achieve satisfactory quality standards. We intend to refine the "Quality"-level annotations standards in future work.

Table 1 Breakdown of annotation quality

\begin{tabular}{ll}
\hline Annotation grade & Percentage \\
\hline High Quality & $61.82 \%$ \\
Quality & $10.91 \%$ \\
Poor & $27.27 \%$ \\
\hline
\end{tabular}

\subsection{Characteristics of retained annotations}

As shown in Table 2, we note that there is no obvious correlation between activity type and the percentage of occluded keypoints. This is because self-occlusion generally plays a larger contribution to the total occlusion rate than occlusion from background objects. 
Table 2 Characteristics of annotated videos

\begin{tabular}{llll}
\hline Category & $\mathrm{n}^{\circ}$ clips & $\begin{array}{l}\text { avg. } \mathrm{n}^{\circ} \\
\text { frames } \\
\text { per video }\end{array}$ & $\begin{array}{l}\% \\
\text { occlusion }\end{array}$ \\
\hline Bricklaying & 66 & 43.64 & 27.69 \\
Transporting & 11 & 26.45 & 17.95 \\
Cutting Plate & 44 & 48.84 & 17.78 \\
Nailing & 74 & 37.93 & 21.04 \\
Plastering & 111 & 47.89 & 24.01 \\
Shovelling & 12 & 35.42 & 11.55 \\
Bolting & 44 & 46.59 & 21.57 \\
Drilling & 11 & 18.00 & 23.15 \\
Sawing & 20 & 55.50 & 34.21 \\
\hline
\end{tabular}

Table 3 characterizes the percentage of keypoints that are occluded or truncated (outside of the image). The low proportion of truncated key points indicates that workers are generally fully contained within the images except for their ankles. The globally higher ratio of shoulder occlusion is due to the posture of the worker remaining globally constant during videos and workers often been seen side-on which perpetuates any self-occlusion of immobile limbs present in the initial frame. However, most worker actions are performed primarily with the arms, wrists and elbows which only self-occlude temporarily.

Table 3 Occluded and truncated ratios of skeleton keypoints

\begin{tabular}{lll}
\hline Keypoint & \% occlusion & \% truncation \\
\hline Nose & 0.00 & 0.06 \\
Left shoulder & 32.89 & 1.64 \\
Left elbow & 19.39 & 1.00 \\
Left wrist & 19.78 & 0.33 \\
Right shoulder & 25.98 & 0.93 \\
Right elbow & 23.84 & 0.36 \\
Right wrist & 28.09 & 0.39 \\
Left hip & 18.54 & 0.21 \\
Left knee & 27.06 & 1.08 \\
Left ankle & 21.29 & 2.26 \\
Right hip & 30.53 & 0.69 \\
Right knee & 23.89 & 1.77 \\
Right ankle & 31.75 & 9.69 \\
\hline
\end{tabular}

\subsection{Ease of use and effectiveness of the annotation tool}

An example video can be found at https://www.useloom.com/share/0adaba9e3af74cfaaee68b453 $\underline{431 \mathrm{bd} 32}$ in which a user shows obtention of a complete, high quality annotation of a 50-frame video in approximately 10 minutes. Most annotators were able to achieve a similar annotation speed, finishing their assigned videos in three hours on average. Approximately 1572 man-hours were spent on this annotation task in total. Qualitive feedback suggests that the assignment was reasonable in terms of length and difficulty.

\section{Conclusion and future work}

In this work we introduce an annotation tool and a set of annotations of workers performing various constructionrelated activities. These annotations were obtained by dividing the videos of the activities among 71 annotators who were able to label around 400 videos in a reasonable number of manhours per annotator. In future work, we intend to annotate further properties of these videos, such as viewpoint, posture and start and end times of the activities being performed. These can then be used to benchmark several state-of-the-art pose estimation algorithms to determine domain-specific advantages and pitfalls. This will be accomplished by performing error analysis that will evaluate how robust pose estimation methods perform in the construction setting according to activity type, posture, keypoint occlusion and object size. Given our videos depict workers performing activities belonging to distinct categories, we also intend to investigate whether pose-informed action recognition and segmentation methods are effective at determining and temporally localizing worker activities. We note that our annotation tool can easily be adapted to annotate keypoints of other construction resources by changing the keypoint nature and layout.

\section{Acknowledgements}

The authors would like to thank the participation of all annotators in the annotation exercise as well as Shuai Tang for helpful comments and annotation quality control. The authors are grateful to Jun Yang for sharing her worker video data. This work was supported by the National Science Foundation grant CMMI 1544999. Opinions, finding and conclusions or recommendations expressed in this material are those of the authors and do not necessarily reflect the views of the National Science Foundation.

\section{References}

Asadi K et al. (2018) Real-time image-to-bim registration using perspective alignment for automated construction monitoring. Proceedings of the 2018 Construction Research Congress. ASCE (American Society of Civil Engineers), New Orleans, LA, USA. https://doi.org/10.1061/9780784481264

Bao R et al. (2016) Characterizing construction equipment activities in long video sequences of earthmoving operations via kinematic features. Proceedings of the 2016 Construction Research Congress. ASCE, San Juan, Puerto Rico. pp. 849858.

Castrejón L et al. (2017) Annotating object instances with a polygon-RNN. Proceedings of the 30th IEEE Conference on Computer Vision and Pattern Recognition. Honolulu, HI, USA. https://doi.org/10.1109/CVPR.2017.477

Cheng $\mathrm{T}$ et al. (2011) Performance evaluation of ultra wideband technology for construction resource location tracking in harsh environments. Automation in Construction. https://doi.org/10.1016/j.autcon.2011.05.001 
Gouett $\mathrm{M}$ et al. (2011) activity analysis for direct-work rate improvement in construction. Journal of Construction Engineering and Management. https://doi.org/10.1061/(ASCE)CO.1943-7862.0000375

Ham Y. et al. (2016) Visual monitoring of civil infrastructure systems via camera-equipped Unmanned Aerial Vehicles (UAVs): a review of related works. Visualization in Engineering. https://doi.org/10.1186/s40327-015-0029-z

Hayes B (2003) Computing science: the easiest hard problem. American $\quad$ Scientist $\quad 90(2)$ : $\quad 113-117$ https://doi.org/10.1511/2011.89.106

Hoiem D et al. (2012) Diagnosing error in object detectors. Lecture Notes in Computer Science https://doi.org/10.1007/978-3-642-33712-3_25

Khosrowpour A et al. (2014) Automated worker activity analysis in indoor environments for direct-work rate improvement from long sequences of RGB-D images. Proceedings of the 2014 Construction Research Congress. Atlanta, Georgia, USA. pp. 729-738

Kim $\mathrm{H}$ et al. (2016) Vision-based object-centric safety assessment using fuzzy inference: monitoring struck-by accidents with moving objects. Journal of Computing in Civil Engineering.

5487.0000562

Kim J et al. (2018) Interaction analysis for vision-based activity identification of earthmoving excavators and dump trucks. Automation in Construction. https://doi.org/10.1016/j.autcon.2017.12.016

Konstantinou E and Brilakis I (2016) 3D matching of resource vision tracking trajectories. Proceedings of the 2016 Construction Research Congress. ASCE, San Juan, Puerto Rico. https://doi.org/10.1061/9780784479827.174

Lecun $Y$ et al. (2015) Deep learning. Nature. https://doi.org/10.1038/nature14539

Lin T et al. (2014) Microsoft COCO: common objects in context. Lecture Notes in Computer Science. https://doi.org/10.1007/978-3-319-10602-1_48

Liu K and Golparvar-Fard M (2015) Crowdsourcing construction activity analysis from jobsite video streams. Journal of Construction Engineering and Management. https://doi.org/10.1061/(ASCE)CO.1943-7862.0001010

Liu M et al. (2017) Potential of convolutional neural networkbased $2 \mathrm{~d}$ human pose estimation for on-site activity analysis of construction workers. Journal of Computing in Civil Engineering. https://doi.org/doi:10.1061/9780784407943

Memarzadeh M et al. (2013) Automated 2D detection of construction equipment and workers from site video streams using histograms of oriented gradients and colors. Automation in Construction 32: 24-37. https://doi.org/10.1016/j.autcon.2012.12.002

Monnot J and Williams R (2011) Construction equipment telematics. Journal of Construction Engineering and Management. https://doi.org/10.1061/(ASCE)CO.19437862.0000281 .
Park MW et al. (2015) Hardhat-wearing detection for enhancing on-site safety of construction workers. Journal of Construction Engineering and Management. https://doi.org/10.1061/(ASCE)CO.1943-7862.0000974

Park MW and Brilakis I (2012) Construction worker detection in video frames for initializing vision trackers. Automation in Construction. https://doi.org/10.1016/j.autcon.2012.06.001

Rezazadeh Azar E and McCabe B (2012) Automated visual recognition of dump trucks in construction videos. Journal of Computing in Civil Engineering. https://doi.org/10.1061/(ASCE)CP.1943-5487.0000179

Roberts D and Golparvar-Fard M (2017) Detecting and classifying cranes using camera-equipped uavs for monitoring crane-related safety hazards. Proceedings of the 2017 International Workshop on Computing in Civil Engineering. ASCE, Seattle, WA, USA. pp. 442-449

Roberts D et al. (2018) Vision-based construction activity anal ysis in long video sequences via hidden markov models: experiments on earthmoving operations. Proceedings of the 2018 Construction Research Congress. ASCE, New Orleans, LA, USA. pp. 164-173

Ronchi M and Perona P (2017) Benchmarking and error diagnosis in multi-instance pose estimation. Proceedings of the IEEE International Conference on Computer Vision. https://doi.org/10.1109/ICCV.2017.48

Russakovsky O et al. (2015) ImageNet large scale visual recognition challenge. International Journal of Computer Vision. https://doi.org/10.1007/s11263-015-0816-y

Seo J et al. (2015) Computer vision techniques for construction safety and health monitoring. Advanced Engineering Informatics, 29(2). https://doi.org/10.1016/j.aei.2015.02.001

Soltani M et al. (2017) Skeleton estimation of excavator by detecting its parts. Automation in Construction. https://doi.org/10.1016/j.autcon.2017.06.023

Son $\mathrm{H}$ et al. (2019) Detection of construction workers under varying poses and changing background in image sequences via very deep residual networks. Automation in Construction, 27-38. https://doi.org/10.1016/j.autcon.2018.11.033

Tang S and Golparvar-Fard M (2017) Joint reasoning of visual and text data for safety hazard recognition. Proceedings of the 2017 International Workshop on Computing in Civil Engineering. ASCE, Seattle, WA, USA. pp. 450-457

Vondrick C et al. (2013) Efficiently scaling up crowdsourced video annotation: a set of best practices for high quality, economical video labeling. International Journal of Computer Vision. https://doi.org/10.1007/s11263-012-0564-1

Wang $\mathrm{H}$ et al. (2011) Action recognition by dense trajectories. Proceedings of the IEEE Computer Society Conference on Computer Vision and Pattern Recognition. https://doi.org/10.1109/CVPR.2011.5995407

$\mathrm{Xu} \mathrm{J}$ and Yoon HS (2019) Vision-based estimation of excavator manipulator pose for automated grading control. Automation in Construction 122-131. https://doi.org/10.1016/j.autcon.2018.11.022 
Yang $\mathrm{J}$ et al. (2016) Vision-based action recognition of construction workers using dense trajectories. Advanced Engineering

Informatics. https://doi.org/10.1016/j.aei.2016.04.009 